\title{
RELATIVELY UNIFORM CONVERGENCE OF SEQUENCES OF FUNCTIONS *
}

\section{E. W. CHITTENDEN}

E. H. Moore $†$ has introduced the notion of uniform convergence of a sequence of functions relative to a scale function. It is the purpose of this paper to study this type of convergence in the field of functions of a real variable.

1. The following definition of relatively uniform convergence is, for the case of a sequence of functions of a real variable, equivalent to the definition given by Moore.

A sequence $\left\{\mu_{n}\right\}$ of single-valued, real-valued functions $\mu_{n}$ of a variable $p$, ranging over a set $\mathfrak{P}$ of real elements $p$, converges relatively uniformly on $\mathfrak{P}$ in case there exist functions $\theta$ and $\sigma$, defined on $\mathfrak{B}$, and for every $m$ an integer $n_{m}$ (dependent on $m$ ), such that for every $n$ greater than or equal to $n_{m}$ the inequality

$$
m\left|\theta-\mu_{n}\right| \leqq|\sigma| \ddagger
$$

holds for every element $p$ of $\mathfrak{B}$.

The function $\sigma$ of the definition above is called a scale function. The sequence $\left\{\mu_{n}\right\}$ is said to converge uniformly relative to the scale function $\sigma$; or more simply, relatively uniformly.

2. The following propositions are immediate consequences of the definition of relatively uniform convergence. $\S$

I. Uniformity of convergence relative to a constant scale function different from zero is equivalent to uniform convergence.

II. Uniformity relative to $\sigma$ implies uniformity relative to every function $\tau$ such that $|\tau| \bar{\Sigma}|\sigma|$.

* Presented to the Society March 22, 1913.

$\dagger$ A Introduction to a Form of General Analysis, The New Haven Mathematical Colloquium, Yale University Press, New Haven, 1910. This memoir will be cited as I. G. A.

\$ The relation (1) holds identically in $p$. In such cases we follow the usage of Moore (cf. I. G. A., p. 27) and omit the variable.

$\$$ Cf. I. G. A., p. 33, et seq. All propositions and theorems of this paper are stated for sequences of functions. The corresponding propositions for series of functions are readily inferred. 
III. Uniformity as to a function $\sigma$ such that $A \leqq|\sigma| \leqq B$, where $A$ and $B$ are positive, implies uniform convergence.

IV. If a sequence converges uniformly relative to a scale function $\sigma$, but does not converge uniformly, then $\sigma$ is not bounded.

$V$. If a sequence of functions is defined on a class $\mathfrak{B}$ and if $\mathfrak{P}$ may be divided into a finite number of parts such that the sequence converges relatively uniformly on each part, then the sequence converges relatively uniformly on \$.*

VI. If a sequence converges relatively uniformly on $\mathfrak{B}, \mathfrak{B}$ may be divided into a sequence $\left\{\mathfrak{B}_{n}\right\}$ such that no two sets $\mathfrak{P}_{n_{1}}, \mathfrak{P}_{n_{2}}\left(n_{1} \neq n_{2}\right)$ have a common element, and such that on each $\mathfrak{B}_{n}$ the sequence converges uniformly.

3 . The following examples are illustrative of relative uniformity of convergence.

(a) The class $\mathfrak{B}$ is the interval $0 \leqq p \leqq 1$; the sequence $\left\{\mu_{n}\right\}$ such that $\mu_{n}(p)=1 / n p(p \neq 0), \mu_{n}(0)=0$. This sequence does not converge uniformly, but the function $\sigma(p)=1 / p$ is effective as a scale function.

(b) The class $\mathfrak{B}$ is the infinite segment, $1 \leqq p ; \mu_{n}(p)=1 / n p$. The sequence converges uniformly, but satisfies the stronger condition of uniform convergence relative to the scale function $\sigma(p)=1 / p . \dagger$

4. Using the notation of $\S 1$, we denote by $\phi_{n}(p)$ the least upper bound of $\left|\theta(p)-\mu_{n^{\prime}}(p)\right|$ for all $n^{\prime} \geqq n$. If the sequence $\left\{\mu_{n}\right\}$ converges to $\theta$ uniformly, relative to a scale function $\sigma(p)$, then for every $m$ there exists an $n_{m}$ such that the inequality (1) is satisfied for all $n \geqq n_{m}$. Hence we may write, in view of the definition of $\phi_{n}(p)$,

$$
m \phi_{n_{m}}(p) \leqq|\sigma(p)| \text {. }
$$

As an immediate consequence of this result we have the following theorem:

Theorem 1. A necessary and sufficient condition that a sequence $\left\{\mu_{n}\right\}$ of functions $\mu_{n}$ converges relatively uniformly on $\mathfrak{P}$ to a limit function $\theta$ is that there exist a sequence $\left\{n_{m}\right\}$ of positive integers such that the sequence $m \phi_{n_{m}}(p)$ has an upper bound $B(p)$ for every $p$.

5. Let $\mathfrak{P}$ be any interval $(a, b)$, and $(r, q)$ a sub-interval of $\mathfrak{B}$ in which the only point of non-uniform convergence $\nmid$ of a sequence $\left\{\mu_{n}\right\}$ is the end point $q$. The sequence $\left\{\mu_{n}\right\}$ being supposed convergent on $(r, q)$, we have a function $A(p) \geqq 1$ such that for every $p$ in $(r, q)$

*Cf. $\$ 6$ of this paper, proposition VIII, which is an extension of proposition $\mathrm{V}$ and the converse of VI.

† Other examples are given later in this paper. Cf. also I. G. A.

$\ddagger$ A point $q$ is a point of non-uniform convergence in case the measure of non-uniformity of convergence of the sequence is greater than zero at $q$. Cf. W. H. Young, P r o c e e d in $\mathrm{g} s$ of the Lond on M a the matical S oc i e t y, ser. 2, vol. I (1903-4) p. 91; also E. W. Hobson, Theory of Functions of a Real Variable, Cambridge University Press, p. 474, \&342; p. $484, \S 349$. 


$$
\left|\theta(p)-\mu_{n}(p)\right| \leqq A(p) .
$$

We will now show that the sequence $\left\{\mu_{n}\right\}$ converges uniformly on $(r, q)$ relative to the scale function $\sigma(p)=A(p) /(q-p) ; \sigma(q)=A(q)$. For each $m$ greater than $1 /(q-p)$ choose $p_{m}$ so that $q-p_{m}=1 / m$. Then in the interval $\left(r, p_{m}\right)$, which contains no point of non-uniformity, the sequence $\left\{\mu_{n}\right\}$ converges uniformly, and therefore $n_{m}$ exists so that for every $n \bar{\gtrless} n_{m}, p$ in $\left(r, p_{m}\right)$, and $p=q$,

$$
m\left|\theta(p)-\mu_{n}(p)\right| \leqq 1 .
$$

But for every $n$, and $p$ in the segment $p_{m} \leqq p<q$,

$$
m\left|\theta(p)-\mu_{n}(p)\right| \leqq A(p) /(q-p) .
$$

The combination of the last two inequalities gives the desired convergence relative to $\sigma$.

The result just obtained is stated in the following theorem:

Theorem 2. If $q$ is on the left an isolated point of non-uniformity of convergence of a convergent sequence of functions, there exists a left neighborhood of $q$ on which the sequence converges relatively uniformly.

A similar statement holds if $q$ is isolated on the right.

6. We have occasion to use the following two propositions from general analysis.*

VII. If a sequence of functions is defined on an enumerable set $\mathfrak{P}$ and converges on $\mathfrak{B}$ it converges relatively uniformly on $\mathfrak{P}$.

VIII. If a sequence of functions is defined and converges relatively uniformly on each of a sequence of classes $\mathfrak{P}_{n}$ it converges relatively uniformly on the class $\mathfrak{B}$ of all elements which are in some class $\mathfrak{P}_{n}$, the least common superclass of the classes $\mathfrak{P}_{n}$.

7. Denote by $\mathfrak{Q}$ the set of all points $q$ of non-uniform convergene of a sequence of functions converging on an interval $\mathfrak{P}$ to a definite limit function; by $\mathfrak{Q}^{\prime}$ the derived set of $\mathfrak{Q}$, and by $\mathfrak{Q}^{\circ}$ the aggregate of the points of $\mathfrak{Q}$ and $\mathfrak{Q}^{\prime} . \mathfrak{Q}^{\circ}$ is closed. The set complementary to $\mathfrak{Q}^{\circ}$ consists of the interior points of an enumerable set of non-overlapping intervals, in each of which the sequence of functions converges relatively uniformly (Theorem 2). It follows from proposition VIII that the sequence converges relatively uniformly on the complement of $\mathfrak{Q}^{\circ}$. Since the points of $\mathfrak{Q}^{\circ}$ which are not in $\mathfrak{Q}^{\prime}$ form an enumerable set, we may state, in view of the preceding remarks and propositions V and VII, the following theorem:

Theorem 3. A necessary and sufficient condition that a sequence of functions converges relatively uniformly on an interval $\mathfrak{P}$ is that it converges relatively uni-

\footnotetext{
* Proposition VII is from I. G. A., p. 87. A simple proof may be given following the lines of the proof of Theorem 2 above. Proposition VIII is an immediate extension of VII.

Trans. Amor. Math. Soc. 14
} 
formly on the derived set $\mathfrak{Q}^{\prime}$ of the set $\mathfrak{Q}$ of the points of $\mathfrak{P}$ which are points of non-uniformity.

COROLLARY I. If $\mathfrak{Q}^{\prime}$ is enumerable the sequence converges relatively uniformly on $\mathfrak{\Re}$.

COROLLARY II. If a sequence of functions does not converge relatively uniformly on $\mathfrak{P}$, the corresponding set $\mathfrak{Q}^{\prime}$ is not enumerable, that is, $\mathfrak{Q}$ is dense on a perfect set.

8. The converse of corollary II above is not true, as is shown by the following example.* $\mathfrak{B}$ is the interval $(0,1)$. We define the sequence $\left\{\mu_{n}\right\}$ as follows: $\mu_{n}(p)=0$, if $p$ is irrational, zero, or equal to $m / k$ ( $m$ and $k$ relatively prime, and $k \neq n) ; \mu_{n}(p)=1$, if $p=m / n(m, n$ relatively prime). For this sequence of functions every $p$ is a point of non-uniformity of convergence. However, the functions of the sequence are all zero on the irrational points. Hence by propositions V and VII the sequence converges relatively uniformly.

Osgood $\dagger$ has given an example of a sequence of continuous functions converging to zero as a limit for which the set $\mathfrak{Q}$ and its derivative form a perfect set, but which converges relatively uniformly because the sequence is identically zero on $\mathfrak{Q}$ and $\mathfrak{Q}^{\prime}$.

9. For sequences of continuous functions we have the following theorem:

THEOREM 4. If a sequence of continuous functions converges on an interval $\mathfrak{B}$ to a continuous limit in such a way that the set $\mathfrak{Q}$ of all points of non-uniformity of convergence is dense on $\mathfrak{B}$, the sequence docs not converge relatively uniformly.

To establish this theorem it is sufficient to show that for every sequence $\left\{n_{m}\right\}$ of positive integers $n_{m}$ there exists a $p$ determined by the sequence $\left\{n_{m}\right\}$ such that the sequence $\left\{m \phi_{n_{m}}(p)\right\} \ddagger$ is not bounded. Theorem 4 follows in view of Theorem 1 .

Let $\left\{n_{m}\right\}$ be any sequence of integers with index $m$, and $\left\{\mu_{n}\right\}$ denote the sequence of continuous functions of the theorem, converging to a continuous limit $\theta$. Since the sequence $\left\{\mu_{n}\right\}$ does not converge uniformly in any subinterval of $\mathfrak{P}$ there exists an integer $m_{1}$ with the following property: for every $N$ there exists an $n_{1}$ greater than $N$ and a $p_{1}$ (interior to $\mathfrak{B}$ ) such that

$$
m_{1}\left|\theta\left(p_{1}\right)-\mu_{n_{1}}\left(p_{1}\right)\right|>1 \text {. }
$$

Since $\theta$ and $\mu_{n_{1}}$ are continuous, we may find an interval $I_{1}$ interior to $\mathfrak{B}$ such that for every $p$ in $I_{1}$

$$
m_{1}\left|\theta(p)-\mu_{n_{1}}(p)\right|>1 \text {. }
$$

${ }^{*}$ Cf. W. H. Young, Proceedings of the London Mathematical Soc i e t y, Ser. 2, vol. I (1903-4), p. 94; also E. W. Hobson, Theory of Functions of a Real Variable, p. 487.

$\dagger$ Non-Uniform Convergence and the Integration of Series Term by Term, A meric a $\mathrm{n}$ Journal of Mathematics, vol. 19 (1897), p. 168.

$\ddagger$ Cf. $\$ 4$ for explanation of this notation. 
Consequently, if we take $N$ equal to $n_{m_{1}}$ of the sequence $\left\{n_{m}\right\}$, we have

for every $p$ in $I_{1}$.

$$
m_{1} \phi_{n_{m_{1}}}(p)>1
$$

By similar reasoning we may show that there exists an $m_{2}$ and an interval $I_{2}$ interior to $I_{1}$ such for every $p$ in $I_{2}$

$$
m_{2} \phi_{n_{m_{2}}}(p)>2 \text {. }
$$

Proceeding step by step in the manner indicated we may obtain a sequence $\left\{m_{j}\right\}$ and a sequence $\left\{I_{j}\right\}$ of closed intervals, each interior to the preceding, such that for every $j$ we have on $I_{j}$

$$
m_{j} \phi_{n_{m_{j}}}(p)>j \text {. }
$$

There must exist an element $p^{\circ}$ common to the intervals $I_{j}$. For such an element we have for every $j$

$$
m_{j} \phi_{n_{m_{j}}}\left(p^{\circ}\right)>j .
$$

The sequence $m \phi_{n_{m}}\left(p^{\circ}\right)$ is therefore unbounded, which was to be proved.

Osgood* has given an example of a sequence of continuous functions converging to a continuous limit for which the set $\mathfrak{Q}$ is dense on $\mathfrak{B}$. It follows from the theorem just proved that this sequence does not converge relatively uniformly.

10. A point of non-uniformity of convergence of a sequence of functions relative to a subset $\Re$ of $\mathfrak{P}$ is a point whose measure of non-uniformity of convergence relative to $\Re$ is greater than zero. $\dagger$

Evidently a point of non-uniformity of convergence relative to $\Re$ is a point of non-uniformity of convergence relative to any set containing $\Re$, and therefore of $\mathfrak{B}$.

A slight extension of the proof of Theorem 4 serves to establish the following generalization of that theorem.

Theorem 5. If a sequence of functions defined on $\mathfrak{P}$ and continuous on $\Re$, a perfect subset of $\mathfrak{P}$, converges on $\mathfrak{P}$ to a limit which is continuous on $\Re$ in such a fashion that the class $\mathfrak{Q}$ of all points of $\Re$ which are points of non-uniformity of convergence relative to $\Re$ is dense on $\Re$, then the sequence of functions does not converge relatively uniformly on $\Re$ or on $\mathfrak{B}$.

Urbana, Ill., May 7, 1913.

*. Osgood, loc. cit., p. 171

† Hobson, loc. cit., p. 484, § 349. 